\title{
Pulsed low-dose RANKL as a potential therapeutic for postmenopausal osteoporosis
}

\author{
Anna Cline-Smith, ${ }^{1}$ Jesse Gibbs, ${ }^{2}$ Elena Shashkova, ${ }^{1}$ Zachary S. Buchwald, ${ }^{1}$ Deborah V. Novack, ${ }^{2}$ \\ and Rajeev Aurora ${ }^{1}$ \\ 'Department of Molecular Microbiology and Immunology, St. Louis University School of Medicine, St. Louis, Missouri, USA. \\ ${ }^{2}$ Division of Bone and Mineral Diseases, Department of Medicine, Washington University in St. Louis, \\ St. Louis, Missouri, USA.
}

\begin{abstract}
A number of studies in model animal systems and in the clinic have established that RANKL promotes bone resorption. Paradoxically, we found that pulsing ovariectomized mice with lowdose RANKL suppressed bone resorption, decreased the levels of proinflammatory effector $T$ cells and led to increased bone mass. This effect of RANKL is mediated through the induction of FoxP3 ${ }^{+} \mathrm{CD}^{25} 5^{+}$regulatory $\mathrm{CD8}{ }^{+} \mathrm{T}$ cells $\left(\mathrm{TC}_{\mathrm{REC}}\right)$ by osteoclasts. Here, we show that pulses of low-dose RANKL are needed to induce $\mathrm{Tc}_{\mathrm{REC}}$, as continuous infusion of identical doses of RANKL by pump did not induce $\mathrm{Tc}_{\mathrm{REC}}$. We also show that low-dose RANKL can induce $\mathrm{Tc}_{\mathrm{REC}}$ at 2, 3, 6, and 10 weeks after ovariectomy. Our results show that low-dose RANKL treatment in ovariectomized mice is optimal at once-per-month doses to maintain the bone mass. Finally, we found that treatment of ovariectomized mice with the Cathepsin $\mathrm{K}$ inhibitor odanacatib also blocked $\mathrm{Tc}_{\mathrm{REC}}$ induction by low-dose RANKL. We interpret this result to indicate that antigens presented to $C D 8^{+} T$ cells by osteoclasts are derived from the bone protein matrix because Cathepsin $\mathrm{K}$ degrades collagen in the bone. Taken together, our studies provide a basis for using low-dose RANKL as a potential therapeutic for postmenopausal osteoporosis.
\end{abstract}

Conflict of Interest: ZSB and RA are listed as inventors on two patents on the use of $\mathrm{TC}_{\mathrm{REC}}$ (9144599) and the use of pulsed low-dose RANKL (pending) as therapy.

Submitted: May 27, 2016 Accepted: July 19, 2016 Published: August 18, 2016

Reference information: JCI Insight. 2016;1(13):e88839 doi:10.1172/jii.insight.88839.

\section{Introduction}

Postmenopausal osteoporosis is a relatively common skeletal condition - affecting $50 \%$ of women over the age of 45 - that leads to bone fractures and disability. Declining estrogen levels, due to loss of ovarian function, results in increased bone resorption and, to a lesser extent, increased bone formation, leading to a net bone loss (1). In addition to loss of estrogen, many genetic and environmental (or nonheritable) factors also modulate the impact of estrogen deficiency on the bone. In mice, the effects of estrogen depletion can be modeled by ovariectomy (OVX). At the cellular level, estrogen deficiency leads to an increased number of osteoclasts due to increased RANKL (2-4) and increased lifespan of the osteoclasts (5-7). In addition, proinflammatory cytokines TNF $\alpha$ and IL-17A expressed by $\mathrm{T}$ cells also promote osteoclastogenesis and resorption activity (8-11). A key mechanism by which $\mathrm{TNF} \alpha$ promotes bone resorption is increasing the sensitivity of osteoclast precursors to RANKL activity $(12,13)$. The critical role of RANKL in driving bone erosion in postmenopausal women has been clinically validated by RANKL blockade, which was accomplished by the use of Denosumab, shown to reduce the risk of fracture in women with osteoporosis (14).

We have previously shown that osteoclasts, in addition to their bone resorption function, also have an antigen presentation activity (15). Osteoclasts produce chemokines that recruit $\mathrm{T}$ cells and, in mice, activate $\mathrm{CD}^{+} \mathrm{T}$ cells. Osteoclasts generated from monocytes isolated from human peripheral blood also showed antigen-presentation activity that could activate both $\mathrm{CD} 4^{+}$and $\mathrm{CD} 8^{+} \mathrm{T}$ cells (16). Murine osteoclasts express only MHC class I at homeostasis on their cell surface and thus activate $\mathrm{CD} 8^{+} \mathrm{T}$ cells. Our laboratory showed that $\mathrm{CD} 8^{+} \mathrm{T}$ cells activated by osteoclasts express the high-affinity IL-2 receptor CD25 and the transcription factor Forkhead box P3 (FoxP3). FoxP $3{ }^{+} \mathrm{CD} 25^{+} \mathrm{CD} 8^{+} \mathrm{T}$ cells are produced in the thymus that have demonstrated immunosuppressive activity (17), and these cells have also been shown to be produced in the periphery. In keeping with the recommendations for nomenclature (18), we refer to the $\mathrm{CD}_{25}{ }^{+} \mathrm{FoxP}^{+}$regulatory $\mathrm{CD} 8^{+} \mathrm{T}$ cells as $\mathrm{Tc}_{\mathrm{REG}}$ and to the $\mathrm{BM}$ and in vitro osteoclast-induced regulatory $\mathrm{CD}^{+} \mathrm{T}$ cells as $\mathrm{OC}-\mathrm{iTc} \mathrm{REG}_{\mathrm{REG}}$. FoxP3 is a master regulator for the development of Tregs that is required for 
their development, maintenance, and function $(19,20)$. FoxP3 has been primarily studied in the context of $\mathrm{CD}^{+} \mathrm{T}$ cells $\left(\mathrm{T}_{\mathrm{REG}}\right)$. Although the $\mathrm{CD} 4^{+} \mathrm{T}_{\mathrm{REG}}$ and $\mathrm{CD} 8^{+} \mathrm{Tc}_{\mathrm{REG}}$ share some common features, $\mathrm{T}_{\mathrm{REG}}$ are critical dominant-negative regulators of self-reactive $\mathrm{T}$ cells. Genetic ablation of FoxP3 or depletion of $\mathrm{T}_{\mathrm{REG}}$ leads to multiorgan autoimmune syndrome (21-23). In contrast, $\mathrm{Tc}_{\mathrm{REG}}$ apparently do not maintain global suppression of autoreactive T cells (24). Nonetheless, consistent with the expression of FoxP3, we have demonstrated that the $\mathrm{OC}-\mathrm{iTc} \mathrm{R}_{\mathrm{REG}}$ are immunosuppressive. In addition, $\mathrm{OC}-\mathrm{iTc} \mathrm{REG}_{\mathrm{RE}}$ also limit osteoclast resorption activity to form a negative loop (25). The suppression of osteoclastogenesis and actin-ring reorganization in mature osteoclasts is accomplished in large part through secretion of and IL-10 (25-27). $\mathrm{Tc}_{\mathrm{REG}}$ also secrete IL- 6 and display CTLA-4 and RANKL on their cell surface. Therefore, while OC-iTc $\mathrm{REG}_{\mathrm{RE}}$ express both pro- and antiresorptive mediators, or studies show that $\mathrm{TC}_{\mathrm{REG}}$ limit osteoclast activity.

We have shown that antigen presentation by osteoclasts is required for $\mathrm{TC}_{\mathrm{REG}}$ induction $(15,27)$. In addition, we previously demonstrated in vitro that osteoclasts crosspresent antigens; therefore, it is presumed that osteoclasts must be actively resorbing bone to induce $\mathrm{TC}_{\mathrm{REG}}$. In the current work, we also examined the source of antigens using odanacatib, a pharmacological inhibitor of Cathepsin K (CTSK). CTSK is a cysteine protease produced by active osteoclasts that is secreted from the ruffled border into the resorptive pits $(28,29)$. CTSK targets type I collagen primarily (30-32) but most likely also degrades noncollagenous proteins in the bone matrix (33). Selective inhibition of CTSK by odanacatib $(32,34)$ has been shown to reduce markers of bone resorption and increase bone density in OVX nonhuman primates (35). CTSK inhibitors also enhance osteoclast survival (36). In our previous studies using decreasing doses of RANKL administration to mice to activate osteoclasts, we found that administration of low-dose RANKL $(0.125 \mathrm{mg} / \mathrm{kg})$ was sufficient to induce $\mathrm{Tc}_{\mathrm{REG}}$ but did not increase osteoclast activity sufficiently to produce significant bone loss. Furthermore, administration of low-dose RANKL induced $\mathrm{Tc}_{\mathrm{REG}}$ in ovariectomized mice. Inflammatory cytokines, such as $\mathrm{TNF} \alpha$ and IL-17A, suppress $\mathrm{Tc}_{\mathrm{REG}}$ induction, but administration of low-dose RANKL derepressed critical molecules expressed on osteoclasts needed for $\mathrm{Tc}_{\mathrm{REG}}$ induction (37). Induction of $\mathrm{Tc}_{\mathrm{REG}}$ in $\mathrm{OVX}$ mice suppressed bone resorption, reduced the number of osteoclasts, increased bone formation rate, decreased the number proinflammatory $\mathrm{T}$ cells $\left(\mathrm{CD} 45^{+} \mathrm{CD} 3{ }^{+} \mathrm{CD} 44^{+} \mathrm{CD} 62 \mathrm{~L}^{-}\right.$cells). Indeed, administration of low-dose RANKL was bone anabolic (37). These results stating that administration of RANKL is bone protective are ostensibly at odds with the observations that RANKL is proresorptive. Here, we set out to reconcile these paradoxical observations. In addition, we demonstrate, in the preclinical model of OVX mice, that low-dose RANKL is potentially an effective treatment of postmenopausal osteoporosis.

\section{Results}

Induction of $T c_{R E G}$ by low-dose RANKL requires pulsed administration. RANKL promotes osteoclastogenesis and bone resorption activity, and increased levels of RANKL - all other factors being equal should promote resorption. Yet, as predicted by our work on $\mathrm{Tc}_{\mathrm{REG}}$, we found that administration of low-dose RANKL to OVX mice led to decreased bone resorption, fewer proinflammatory effector $\mathrm{T}$ cells $\left(\mathrm{T}_{\mathrm{EFF}}\right)$, and increased bone mass in our previous study (37). The question remains: if there are significant levels of endogenous RANKL already present after menopause, why does administration of additional low levels of RANKL lead to $\mathrm{Tc}_{\mathrm{REG}}$ induction? We hypothesized that the (absolute) levels of RANKL may not be critical; instead, the pulsed delivery of RANKL may be important for this phenomenon. To test the hypothesis, we delivered identical doses of RANKL $(0.25 \mathrm{mg} / \mathrm{kg})$ over 48 hours using either implanted ALZET pumps or injected (twice 24 hours apart) into peritoneal cavity. The mice were ovariectomized, and the pumps containing PBS or RANKL were implanted after 14 days. On days 14 and 15, the mice implanted with pumps containing RANKL were injected with PBS, and mice implanted with pumps containing PBS were injected with RANKL. We found that, in mice in which RANKL was delivered by pumps, no $\mathrm{TC}_{\mathrm{REG}}$ induction was observed (Figure 1A, center, and Figure 1B). In contrast, administering RANKL twice in OVX mice 24 hours apart induced robust levels of $\mathrm{TC}_{\mathrm{REG}}$ (Figure 1A, right, and Figure $1 \mathrm{~B}$ ). Concomitant with the $\mathrm{Tc}_{\mathrm{REG}}$ induction, pulsed administration of low-dose RANKL led to decreased $\mathrm{T}_{\mathrm{EFF}}$ (Figure 1C) and bone resorption (Figure 1D). Analysis of proximal tibias by $\mu \mathrm{CT}$ showed about $69 \% \pm 18 \%$ increased bone mass (BV/TV; Figure $1 \mathrm{E}$ ), increased bone mineral density $(10.4 \% \pm 1.2 \%$; Figure $1 \mathrm{~F})$, and fewer number of osteoclasts (Figure $1 \mathrm{G})$, resulting in improved trabecular parameters (Supplemental Figure 1) in RANKL-pulsed mice. In contrast, continuous delivery of RANKL by ALZET pumps led to no significant change in $\mathrm{T}_{\mathrm{EFF}}$ (Figure 1C), increased bone resorption (Figure 1D), and decreased bone volume (Figure 1E), bone density (Figure 
A

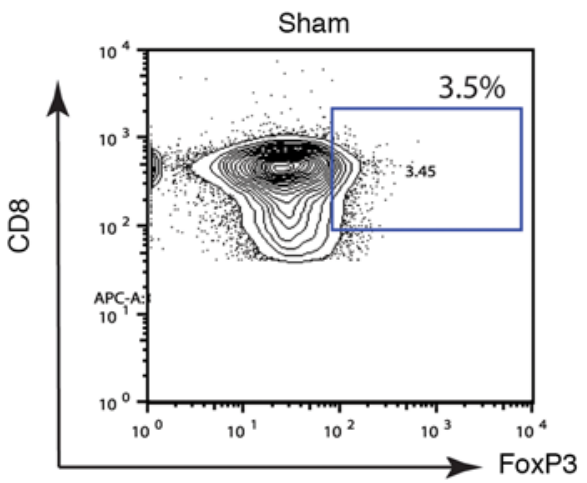

B

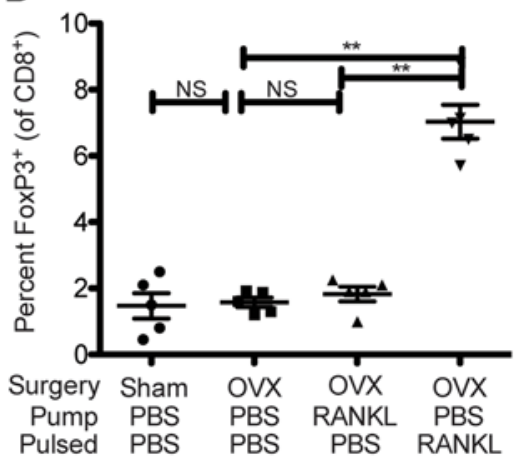

C

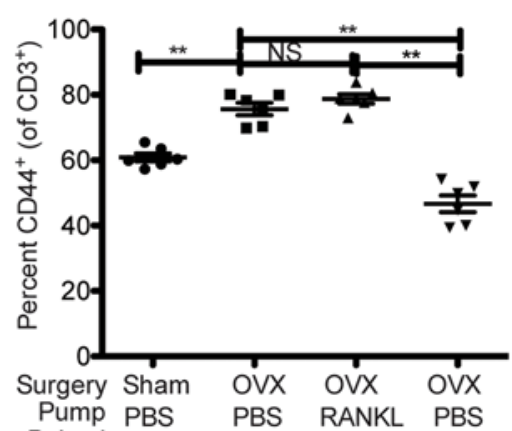

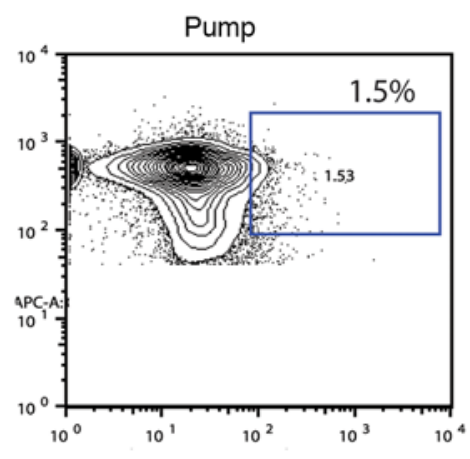

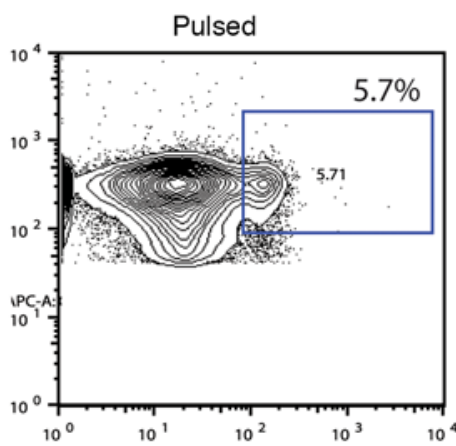

\section{E}
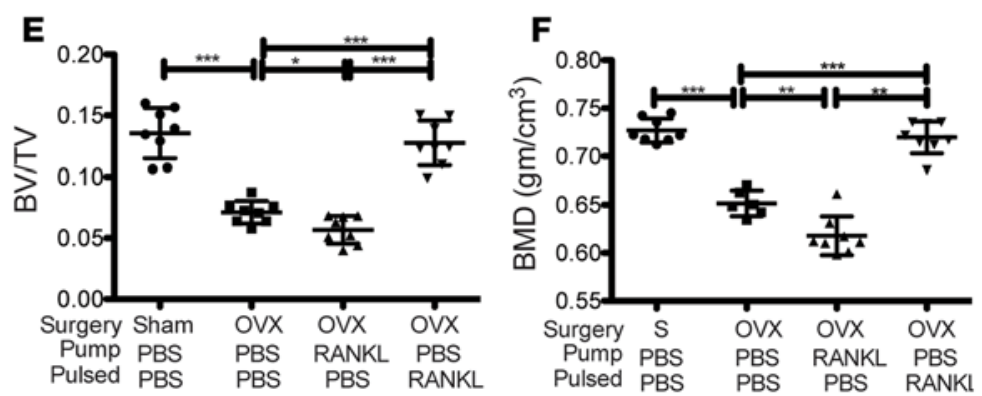

D
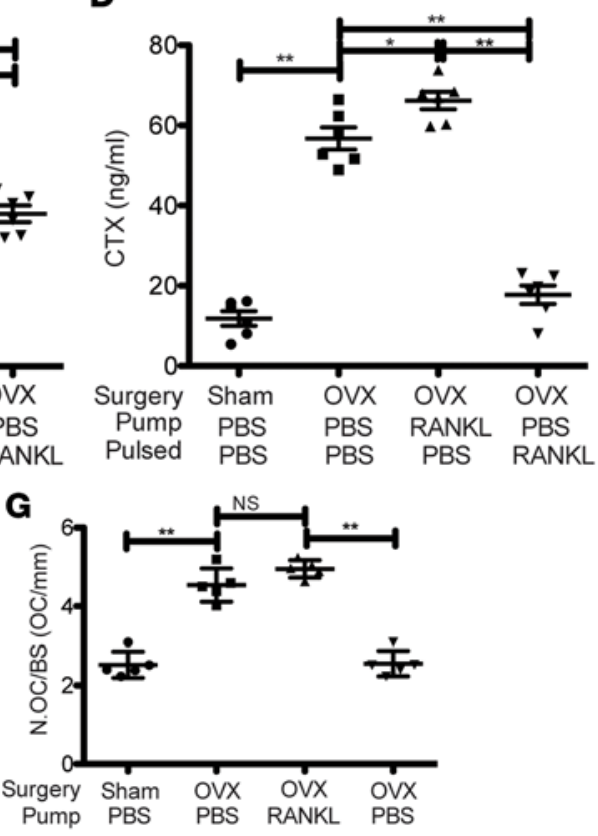

H
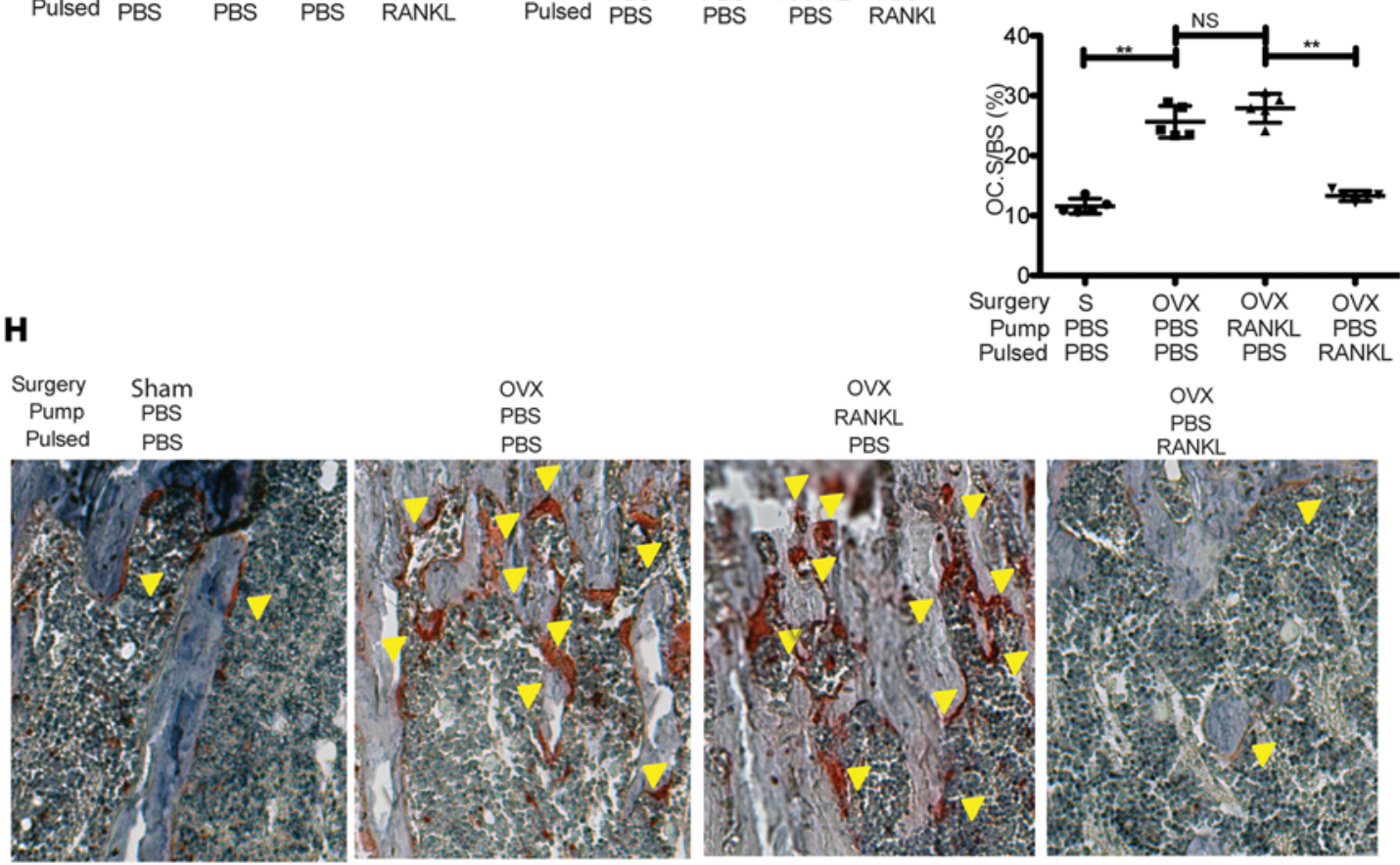

OVX RANKL

OVX

RANKL
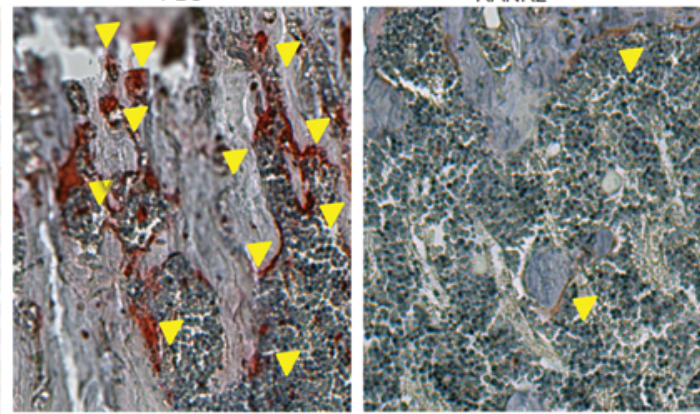
Figure 1. Pulsed RANKL is required for the bone anabolic effect of low-dose RANKL. Twelve-week-old C57BL/6] mice were either sham-operated or OVX using pumps or pulsed with low-dose RANKL. Two weeks after surgery, ALZET pumps were implanted in the i.p. cavity that contained either PBS or RANKL. All groups were pulsed with PBS or with an equivalent dose of RANKL ( $0.125 \mathrm{mg} / \mathrm{kg}$, pulsed twice 24 hours apart) as indicated in the plots.

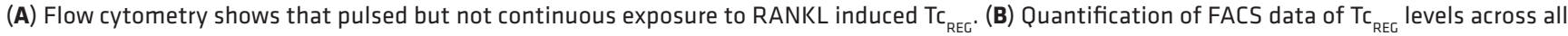
groups (6-8 mice/group). (C) Consistent with $\mathrm{Tc}_{\mathrm{REC}}$ induction, decrease in $\mathrm{T}_{\mathrm{EFF}}$ cells (gated on $\mathrm{CD}_{4} 5^{+} \mathrm{CD} 3^{+}$cells, and then on CD44 $4^{+}$was only observed in mice pulsed with RANKL but not treated with RANKL delivered by pumps. (D) Decreased levels of bone resorption were observed in mice pulsed with low-dose RANKL but not in mice with continuous exposure to RANKL. Modestly higher (but statistically significant) levels of bone resorption were observed in mice treated with continuous RANKL. (E) Bone volume (BV/TV) decreased in mice where RANKL was delivered by pump but was restored to levels observed in sham-operated mice when pulsed with RANKL. (F) Consistent with serum CTX and BV/TV results, an increase in bone mineral density (BMD) was observed in mice treated with pulsed RANKL but decreased in mice where RANKL was delivered by pump. (G and $\mathbf{H})$ Pulsed lowdose RANKL decreased osteoclast numbers. Quantitation of osteoclast numbers/mm bone (N.OC/BS) and osteoclast surface/bone surface (OC.S/BS) is shown in $\mathbf{G}$, and representative images are shown in $\mathbf{H}$ from sham and ovariectomized mice treated with infused and pulsed RANKL. Arrowheads indicate Tartrate-resistant acid phosphatase-positive (TRAP+) cells. Data is from 6-8 mice per group. Group statistical significance was calculated using Mann-Whitney $U$ test. ${ }^{* *} P<1 \times 10^{-3} ;{ }^{* *} P<0.01 ;{ }^{*} P<0.05$.

$1 \mathrm{~F}$ ), number of osteoclasts (Figure $1 \mathrm{G}$ ), and trabecular parameters. These results indicate that pulsed but not continuous delivery of RANKL is required for $\mathrm{Tc}_{\mathrm{REG}}$ induction. Indeed, our results support the notion that increasing RANKL levels by continuous provision leads to bone loss.

$T c_{R E G}$ induction by low-dose RANKL is independent of time after OVX. In all previous experiments, we have treated mice with pulsed low-dose RANKL 2 or 3 weeks after OVX. As menopause is a decline of ovarian function over 1-3 years (38), using a 2-week time point lacks clinical significance. Moreover, as bone loss occurs most rapidly at early times of menopause (1) and after OVX (39), we wanted to test whether low-dose RANKL treatment could induce $\mathrm{Tc}_{\mathrm{REG}}$ at later times after OVX. To this end, mice were treated with low-dose RANKL at various times after OVX and then sacrificed 10 days after treatment to determine response. The observed level of $\mathrm{Tc}_{\mathrm{REG}}$ induction in the $\mathrm{BM}$ was similar, regardless of the time after OVX (Figure 2A). Unexpectedly, there appears to be some periodicity (Figure 2B) in the effectiveness of RANKL-induced $\mathrm{Tc}_{\mathrm{REG}}$ to suppress $\mathrm{T}_{\mathrm{EFF}}$. Nonetheless, there was a decrease in the number of $\mathrm{T}_{\mathrm{EFF}}$ at all time points tested (Figure $2 \mathrm{~B}$ ). As before, bone resorption levels assessed by serum were effectively decreased (Figure 2C). Bone volume (Figure 2D) and bone mineral density (Figure 2E) were all increased relative to untreated mice, regardless of the time after OVX, indicating that low-dose RANKL is effective as bone anabolic treatment at all times tested.

Determining optimal dosing schedule. In previous experiments, we determined that the half-life of adoptive transferred $\mathrm{Tc}_{\mathrm{REG}}$ in the $\mathrm{BM}$ of the OVX mice was approximately 5 days. The bone protective effect of the transferred $\mathrm{TC}_{\mathrm{REG}}$ lasted about 2 half-lives (26). Here, we repeated this experiment; however, we used lowdose RANKL to induce $\mathrm{Tc}_{\mathrm{REG}}$ in situ. Low-dose RANKL was administered 2 weeks after OVX, and bone resorption in sham-operated mice and in treated and untreated OVX mice was monitored using serum C-terminal telopeptide of type 1 collagen (serum CTX) every 5 days. Our data is consistent with previous findings $(1,39)$ that the highest levels of bone resorption are observed in the first 2 weeks after OVX, decrease over the next 10 days in OVX animals, yet remain significantly higher over sham levels (Figure $3 \mathrm{~A}$ ). As seen in Figure 3A, the effect of $\mathrm{Tc}_{\mathrm{REG}}$ induced by low-dose RANKL lasted for about 30 days, as CTX levels start to rise by day 35 after treatment. Consistent with serum CTX and data shown in Figures 1 and 2, increased bone volume was observed in treated mice at days 25 and 35 relative to untreated OVX mice. Therefore, we concluded that treatment every 30 days would be optimal.

To further evaluate the 30-day dosing and to assess whether repeated dosing is effective and tolerated, we treated 4 groups of OVX mice ( 2 weeks after OVX) every 30 days over 90 days. A group of mice was sacrificed at days $10,30,60$, and 90 to obtain tibias for bone volume and density. The data show the lowdose RANKL treatment was effective at reducing bone resorption at all time points, starting at day 10 (Figure 3C). The bone anabolic effect was also observed in these experiments with BV/TV (Figure 3D). These data also show that the levels of bone volume in OVX mice stay constant and are similar to levels in sham-operated mice, even after 3 repeated treatments.

Inflammatory cytokines play an important role in bone loss in postmenopausal osteoporosis $(8$, 40). To assess if $\mathrm{T}$ cells producing proinflammatory cytokines efflux from the BM space, we collected peripheral blood of mice at day 90, lysed the RBC, and then stimulated the cells with plate-bound antiCD3 and anti-CD28 antibodies. Supernatants from the cells were collected at 36 hours, and the cytokine levels were assessed using multiplexed ELISA. Of the 6 cytokines assayed, only TNF $\alpha$ and IL-17A were detected in untreated OVX mice. These cytokines were not detected in OVX mice treated with 
A

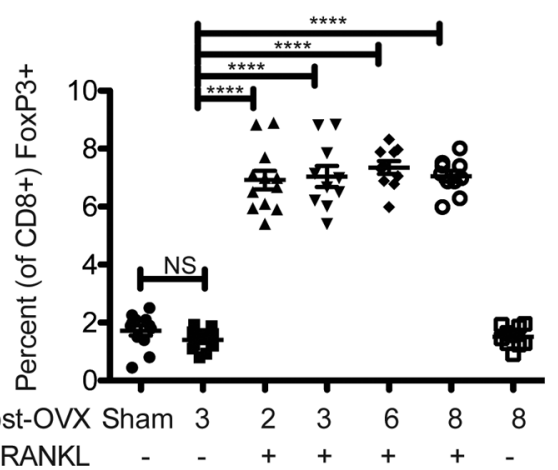

C

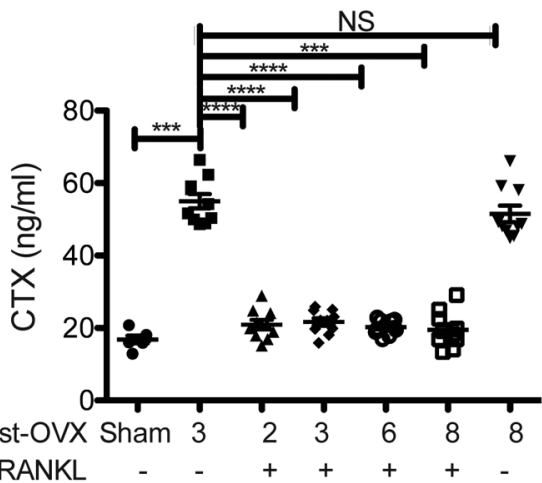

$\mathbf{E}$

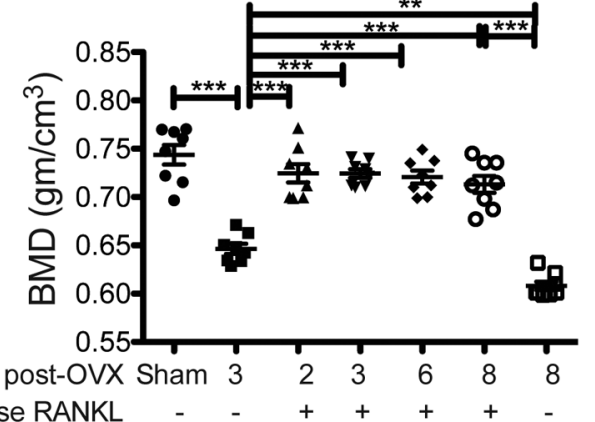

$\begin{array}{lllllll}\text { Weeks post-OVX Sham } & 3 & 2 & 3 & 6 & 8 & 8 \\ \text { L } & - & + & + & + & + & -\end{array}$
B

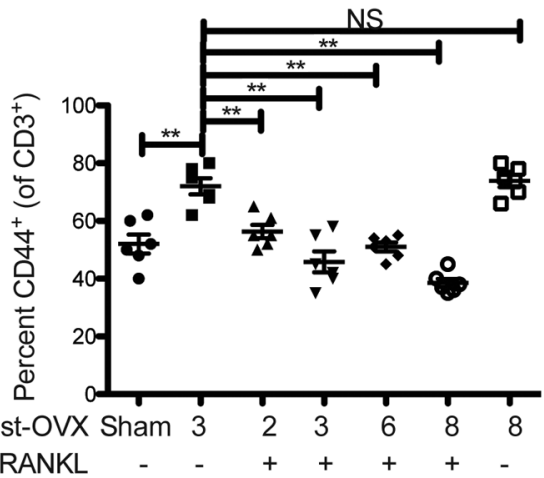

D

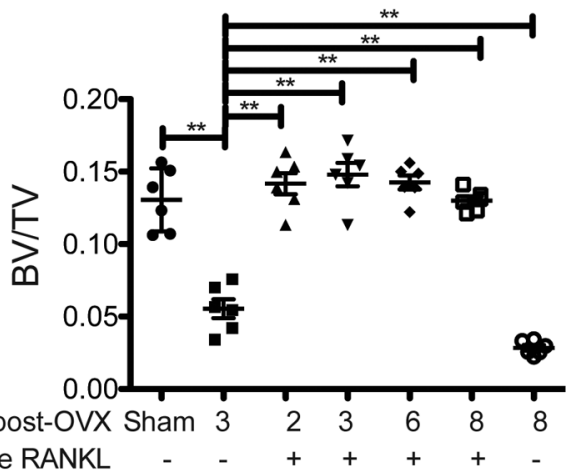

Figure 2. The bone anabolic effect of low-dose RANIKL is independent of time after ovariectomy. Sham surgery or ovariectomy (OVX) was performed on mice at 12 weeks of age. At times (in weeks) indicated after OVX, 2 doses of RANKL $(0.125 \mathrm{mg} / \mathrm{kg}$ ) were administered 24 hours apart. Ten days after first treatment, mice were sacrificed. Low-dose RANKL (A) induced $\mathrm{TC}_{\mathrm{REC}}$ and (B) decreased proinflammatory effector cells in the femora, measured using flow-cytometry. (C) Treatment decreased serum CTX levels. Measurements of proximal tibia by $\mu \mathrm{CT}$ show increased bone mass (BV/ TV) (D) and bone mineral density (BMD) (E) across time course. Data is from 6-12 mice per group. Group statistical significance was calculated using Mann-Whitney $U$ test. ${ }^{* * *} P<1 \times 10^{-4} ;{ }^{* *} P<1 \times 10^{-3} ;{ }^{* *} P<0.01$.

low-dose RANKL but were detectable in mice treated every 30 days with zoledronic acid (Figure $3 \mathrm{E}$ ). These cytokines are only detectable at these levels, with stimulation, at 90 days after treatment. Much lower (and less reliable) levels of cytokines were observed at the earlier time points. Together, these data show that multiple treatments with low-dose RANKL were sufficient to ameliorate OVX-induced osteoporosis and to lower the potential inflammation, an effect that was not attained by the bisphosphonate.

The CTSK inhibitor, odanacatib, blocks $T c_{R E G}$ induction by low-dose RANKL. To determine whether the antiresorptive effect of $\mathrm{Tc}_{\mathrm{REG}}$ induced by low-dose RANKL would be synergistic with inhibition of CTSK, we first administered odanacatib to OVX mice and then treated them with low-dose RANKL. Unexpectedly, we found that pretreating mice with CTSK inhibitor blocked $\mathrm{Tc}_{\mathrm{REG}}$ induction by low-dose RANKL (Figure 4, A and B). To confirm that odanacatib does not adversely affect the CD8 ${ }^{+} \mathrm{T}$ cells, we used antigen-loaded osteoclasts to induce $\mathrm{Tc}_{\mathrm{REG}}$ in culture. As previously described, we cocultured ovalbumin-specific CD8 ${ }^{+} \mathrm{T}$ cells from OT-I mice (41) with peptide-antigen loaded osteoclasts (42). Equal levels of FoxP3 induction were observed in the $\mathrm{CD}^{+} \mathrm{T}$ cells, in the presence or absence of odanacatib (Figure 4C). We interpret this result to indicate that the CTSK inhibitor does not affect $\mathrm{CD}^{+} \mathrm{T}$ cells directly or suppress FoxP3 induction. Indeed, these results support the idea that actively resorbing osteoclasts are needed for $\mathrm{Tc}_{\mathrm{REG}}$ induction because they are released from the bone matrix. Furthermore, these results indicate that the antigens presented to $\mathrm{CD} 8^{+} \mathrm{T}$ cells must be generated from bone matrix proteins. 

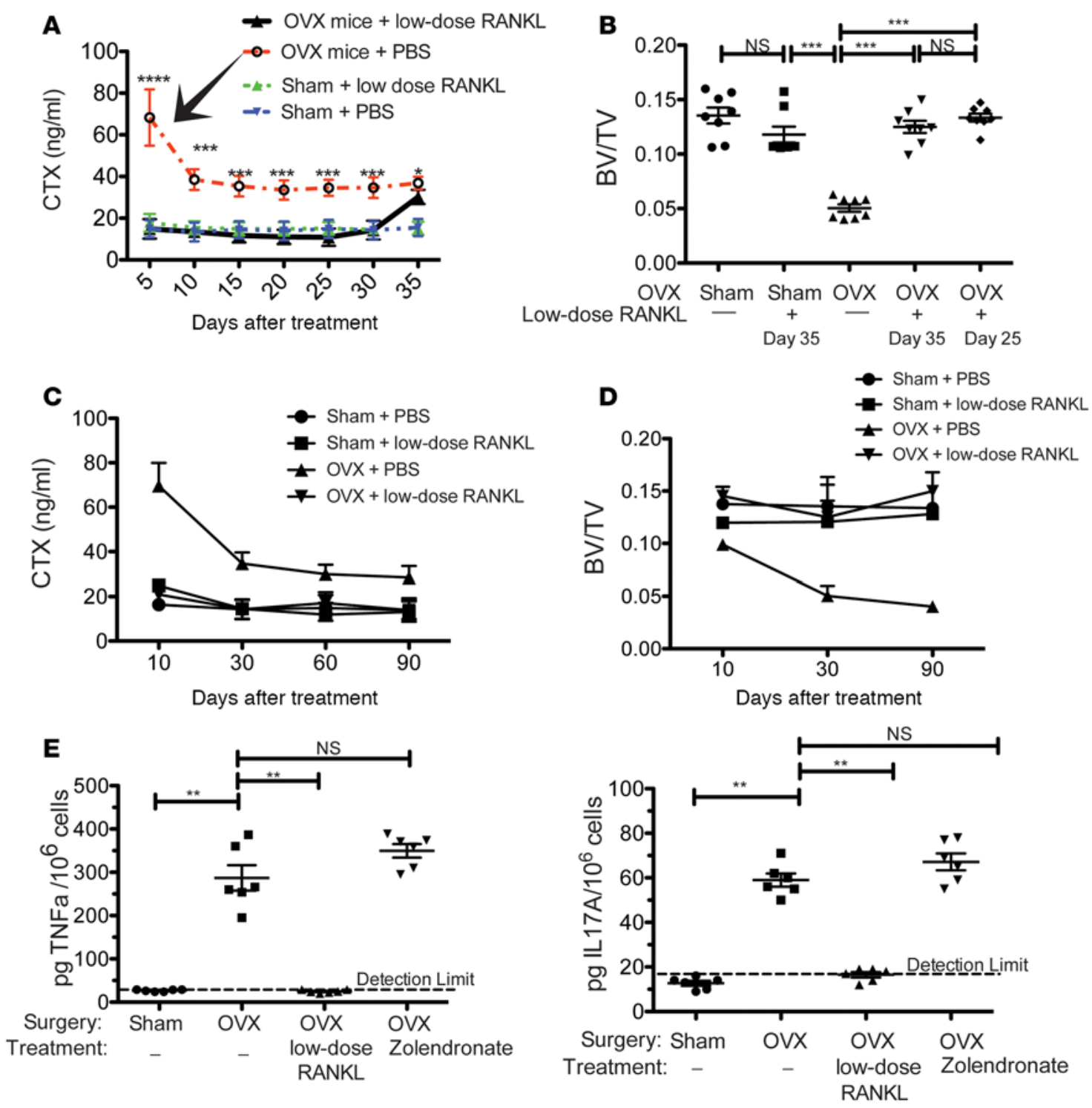

Figure 3. Pulsing RANKL once per month is optimal to limit bone resorption and inflammation. (A) To assess the kinetics of a single treatment, C57BL/6] ovariectomized mice were treated with low-dose RANKL (administered twice 24 hours apart, 2 weeks after OVX). (B)Although serum CTX levels start to rise at day 35, bone mass as assessed by $\mu \mathrm{CT}$ (BV/TV) was relatively stable at days 25 and 35 . (C-E) To assess the effect of repeated dosing, 12-week-old OVX mice then treated every 30 days over a span of 90 days. (C) Serum CTX levels for each of the 4 groups. (D) Representative animals were sacrificed (5-7 mice/group), and BV/TV of the proximal tibias is shown. (E) Cells that secrete TNF $\alpha$ and IL-17A could be detected in peripheral blood 120 days after OVX (6 mice/group). Multiplexed ELISA used media collected after 30 hours of culturing to quantify cytokines. Groups were ovariectomized and treated with low-dose RANKL or zoledronic acid. Group statistical significance was calculated using Mann-Whitney $U$ test. ${ }^{* * * *} P<1 \times 10^{-4} ;{ }^{* * *} P<1 \times 10^{-3} ;{ }^{* *} P \leq 0.01,{ }^{*} P<0.05$.

\section{Discussion}

The proresorptive effects of RANKL, through increasing osteoclastogenesis and bone resorption functions in bone erosion diseases, are well documented. Postmenopausal bone loss is regarded as an inflammatory condition because of the causal role of proinflammatory cytokines produced by $\mathrm{T}_{\mathrm{EFF}}$ (43). Therefore, rather than focusing on either system alone, restoring homeostasis in both the cells that regulate bone mass and immune mediators should be superior. In the current study, we demonstrate that, surprisingly, low-dose RANKL when delivered as a pulse - is efficient at ameliorating OVX-induced osteoporosis. In contrast, continuous delivery of the identical dose of RANKL over the same time period leads to increased bone resorption. These results can only be explained in the context of the negative feedback loop between osteoclasts and $\mathrm{CD} 8^{+} \mathrm{T}$ cells.

Our laboratory was the first to our knowledge to identify that osteoclasts act as antigen-presenting cells that recruit $\mathrm{CD} 8^{+} \mathrm{T}$ cells and induce FoxP3 and CD25 in the CD8 ${ }^{+} \mathrm{T}$ cells. Although such 
A

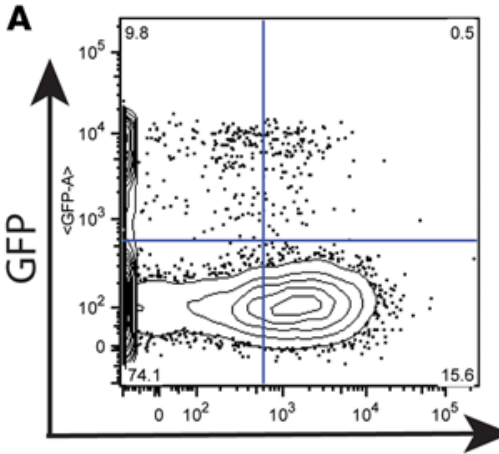

-RANK

+Odanacatib

B

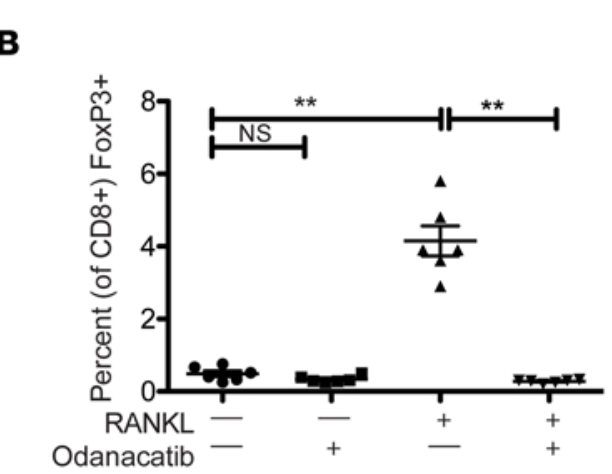

+RANKL

-Odanacatib

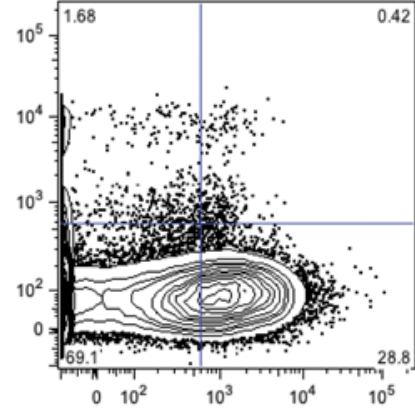

+RANKL +Odanacatib

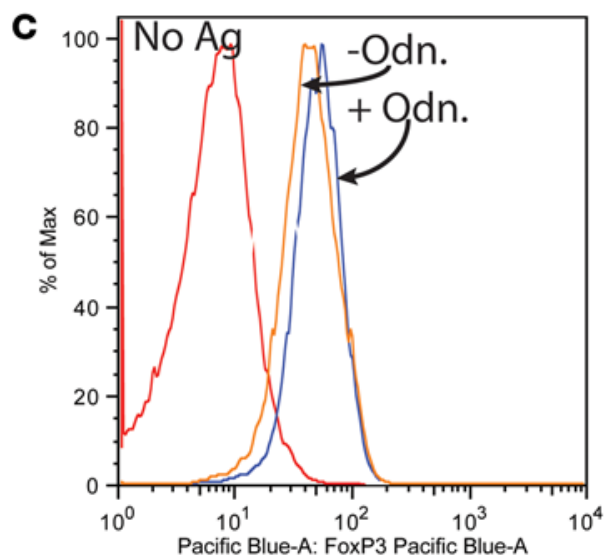

Figure 4. Active antigen processing by osteoclasts is required for $\mathrm{TC}_{\mathrm{REC}}$ induction. (A) FoxP3 ${ }^{\text {eCFP }}$ reporter mice were treated with odanacatib (Odn), and then low-dose RANKL was administered. In the presence of Odn, $\mathrm{Tc}_{\mathrm{REC}}$ (GFP+CD8+ cells) induction was not observed (right panel). (B) The results are quantified from 6 animals/group. (C) In culture, BM cell-derived osteoclasts are able to induce FoxP3 in OVA-specific OT-I CD8 ${ }^{+} \mathrm{T}$ cells when pulsed with peptide antigen (SIINFEKL).

cells have been documented in mice and humans, they have not been well studied, in part due to their very low abundance in lymphoid tissue. In the context of OVX-induced osteoporosis, our previous studies showed that proinflammatory cytokines, specifically TNF $\alpha$ and IL-17A, transcriptionally repress the Notch ligand DLL4 in osteoclasts. $\mathrm{TC}_{\mathrm{REG}}$ cannot be induced in the presence of $\mathrm{TNF} \alpha$ or IL-17A because DLL4 is required for $\mathrm{TC}_{\mathrm{REG}}$ induction by osteoclasts. Pulsed RANKL de-represses DLL4 expression and restores $\mathrm{Tc}_{\mathrm{REG}}$ induction, which reduces $\mathrm{T}_{\mathrm{EFF}}$ and bone resorption and increases bone formation (37).

While our previous studies established a mechanism for the regulation of $\mathrm{Tc}_{\mathrm{REG}}$ induction, a number of questions remained unanswered: first, in estrogen-depleted animals, if there are abundant levels of RANKL present, why does administration of a small amount RANKL activate osteoclasts to induce $\mathrm{Tc}_{\mathrm{REG}}$, while endogenous RANKL does not? One possible explanation is that the molecules (e.g., RANKL, osteoprotegerin [OPG], and other cytokines) that are signals between cells are in such exquisite balance that even small changes in RANKL concentration are sensed to generate a response. Alternatively, but not mutually exclusive, pulsed delivery of low-dose RANKL generates a different response in osteoclasts than that generated by changes in the endogenous RANKL concentration. To test this notion, we used pumps to continuously deliver low-dose RANKL. Our results (Figure 1) show that, when RANKL was delivered continuously by pump, OVX mice lost more bone than untreated mice. In contrast, when the same cumulative amount of RANKL was delivered in 2 doses 24 hours apart, a bone anabolic activity was observed. As pulsing is required for $\mathrm{Tc}_{\mathrm{REG}}$ induction, this ostensibly resolves the paradox of how low-dose RANKL can target osteoclasts differently: when provided continuously, it promotes resorption, and when pulsed, it is antiresorptive through induction of $\mathrm{Tc}_{\mathrm{REG}}$. Notably, parathyroid hormone $(\mathrm{PTH})$ targets osteoblasts sim- 
ilarly: it is bone anabolic due to increased osteoblastogenesis when delivered intermittently but promotes RANKL production to induce bone loss when delivered continuously (44).

The experiments with odanacatib also highlight two additional features: first, because PTH targets osteoblasts, it synergizes with bone resorption inhibitors that target CTSK or with bisphosphonates (45). In contrast, odanacatib (Figure 4) and bisphosphonates (37) block $\mathrm{Tc}_{\mathrm{REG}}$ induction. This experiment provides further evidence that active osteoclasts are needed for $\mathrm{Tc}_{\mathrm{REG}}$ induction. We expect that low-dose RANKL will be synergistic with intermittent PTH as they target different cells. Furthermore, osteoclasts display the unusual activity of cross-presentation of antigens. As CTSK targets the dissolution of the protein matrix, but does not inactivate the osteoclasts like zoledronate, we interpret the lack of $\mathrm{Tc}_{\mathrm{REG}}$ induction in the presence of odanacatib to also indicate that the antigens presented to $\mathrm{CD} 8^{+} \mathrm{T}$ cells are generated from the bone proteins and not from the osteoclast-endogenous proteins.

To assess the therapeutic potential of low-dose RANKL for postmenopausal osteoporosis, our study tested the effectiveness after OVX. We found that low-dose RANKL was effective at all time points tested up to 8 weeks after OVX (Figure 2). Furthermore, we determined the optimal dosing schedule for OVX mice. A single treatment ( 2 administrations, 24 hours apart) was effective at suppressing bone resorption and restoring bone mass for over 30 days (Figure 3). As the administered RANKL is most likely cleared in less than 24 hours, the effect is surprisingly long lasting. We have estimated, based on sampling BM

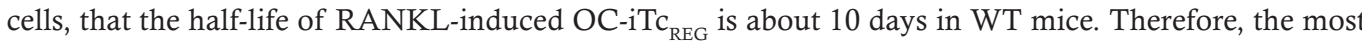
likely explanation of the length of effect is a combination of 2 consequences. First, OC-iTc ${ }_{\mathrm{REG}}$ reduced osteoclast numbers (Figure 1); restoration of osteoclast numbers to pretreatment levels in estrogen-depleted animals takes time. Second, $\mathrm{OC}-\mathrm{iTc} \mathrm{REG}_{\mathrm{RE}}$ also suppressed the levels of proinflammatory T cells (Figure 1), which drive bone resorption through secreted cytokines like TNF $\alpha$ and IL-17A (Figure 3). These cytokines promote osteoclastogenesis (9) and, along with FasL $(5,6,46)$, are responsible for tipping the balance toward net bone loss. Together, the reductions in osteoclast numbers and in the proinflammatory cytokines are most likely responsible for the length of effect observed. Repeated monthly dosing was also effective in maintaining bone mass over the 90 days. In addition to maintaining bone mass, our results show that low-dose RANKL reduced levels of proinflammatory $\mathrm{T}_{\mathrm{EFF}}$. These $\mathrm{T}_{\mathrm{EFF}}$ are detected in the $\mathrm{BM}$ at earlier time points but could be detected in peripheral blood at 90 days in untreated mice (Figure 3). These T cells producing TNF $\alpha$, IL-6, and IL-17A have been observed in postmenopausal osteoporotic women (47) and may contribute to other comorbidities such as mood disorders, joint pain, and atherosclerosis observed in postmenopausal women.

In conclusion, this study establishes the feasibility of using low-dose RANKL for treatment of osteoporosis in a preclinical animal model. The mechanistic insights provided by this study can be summarized in the following manner. First, pulsed or intermittent delivery of RANKL behaves differently than continuous exposure to RANKL. While continuous delivery of RANKL promotes osteoporosis, pulsed delivery promotes a bone anabolic effect through $\mathrm{Tc}_{\mathrm{REG}}$ induction. Second, inhibition of CTSK by odanacatib prevents $\mathrm{Tc}_{\mathrm{REG}}$ induction in vivo, indicating that bone matrix proteins are most likely the source of the antigens presented by osteoclasts to CD ${ }^{+} \mathrm{T}$ cells. This interpretation is consistent with proteomic studies showing that $90 \%$ of the protein content of bone is type I collagen (48). Furthermore, collagen-specific T cell receptors are known to exist in the repertoire of $\mathrm{T}$ cells in both mice (49-51) and humans (52). Osteoclasts present antigens during bone resorption and induce a regulatory phenotype to prevent autoimmune responses. Finally, we determined that once-per-month dosing was optimal to restore bone mass in OVX mice, and repeated dosing maintained bone mass and reduced circulating $\mathrm{T}_{\mathrm{EFF}}$ in peripheral blood that could potentially produce proinflammatory cytokines. The beneficial effects of the pulsed low-dose RANKL treatment were maintained even when started 8 weeks after OVX, suggesting that this treatment may be effective in the early and later stages after hormone withdrawal.

\section{Methods}

Mice. C57BL/6J mice (model 000664) were purchased from the Jackson Laboratory or used from in-house breeding colonies. Breeders of FoxP3 ${ }^{\mathrm{eGFP}}$ reporter (model 006772) mice were purchased from the Jackson Laboratory and bred in-house for these experiments. OT-I Rag ${ }^{-1-}$ mice were purchased from Taconic.

OVX, pump implantation, and inhibitors. Bilateral OVX was performed on 12- to 14-week-old mice. Mice were anesthetized using $2.5 \%$ isoflurane to initiate anesthesia and $1 \%$ for maintenance. The ovaries were 
accessed through a single incision in the skin and exteriorized through muscle wall on each side. Each ovary was clamped using hemostat and removed by a single cut. Skin staples ( $3 \mathrm{M}$ ) were used to close the skin incision. To minimize discomfort after surgery, $0.025 \mathrm{mg} / \mathrm{kg}$ Buprenorphine was administered s.c.

Pumps were implanted 3 weeks after OVX or sham surgery. Pumps (ALZET, catalog 1003D) were filled as recommended by the supplier with PBS or RANKL solution. To confirm delivery of RANKL to the bone, pumps contained Alzarin red with PBS in some experiments. The pumps were primed by placing them in sterile saline for 18 hours prior to installation. Mice were anesthetized using $2.5 \%$ isoflurane and $1 \%$ for maintenance. Pumps were placed in the peritoneal cavity using a small incision in the skin, positioned using blunt forceps, and closed using sutures. Pumps were recovered and weighed at sacrifice and were compared with weight of full pumps.

Zoledronate (Selleck Chemicals) was administered at $30 \mu \mathrm{g} / \mathrm{kg}$ via tail vein. Odanacatib (Selleck Chemicals) stock solution was prepared in DMSO at $50 \mathrm{mg} / \mathrm{ml}$ and administered at $20 \mathrm{mg} / \mathrm{kg}$ i.p. in a volume of $100 \mu \mathrm{l}$. Due to the low solubility of odanacatib in water, the stock solution was freshly diluted into cyclodextrin (10 mg/ml prepared in PBS, catalog C4680, Sigma-Aldrich). Since the halflife of odanacatib in vivo is 6 hours in rats (34), it was administered twice 24 hours apart at the same time as RANKL.

Antibodies and FACS. The FACS data shown in Figures 1, 3, and 4 are from BM cells. BM cells were isolated from mice sacrificed by $\mathrm{CO}_{2}$ asphyxiation; the long bones were harvested, one end cap of the bone was removed, and the long bones were placed in a $0.7-\mathrm{ml}$ microcentrifuge tube and pierced with a $22 \mathrm{G}$ needle at the bottom of the tube. The $0.7-\mathrm{ml}$ tube was fitted inside a $1.5-\mathrm{ml}$ microcentrifuge tube. The assembly was spun for 30 seconds at $16,000 \mathrm{~g}$. The pellet was resuspended in $1 \mathrm{ml}$ of PBS. Then, RBCs were lysed (PharmLyse; BD Biosciences). For FACS, cells were blocked with anti-mouse FcgRIII/ IIR (catalog 553141, BD Pharmingen) for 10 minutes and then stained for 45 minutes on ice with fluorophore-conjugated antibody. Stained cells were washed, fixed with $2 \%$ paraformaldehyde and analyzed on LSRII instrument with CellQuest (BD Biosciences) software. Data analyses were performed with FlowJo software (version 8.73; Tree Star Inc.). Anti-mouse fluorophore-conjugated antibodies for FACS were: PE-conjugated anti-mouse CD8a (clone 53-6.7; BD Pharmingen), AF700-conjugated anti-mouse CD44 (clone IM7; BD Pharmingen), e450-conjugated anti-mouse FoxP3 (clone FJK-16s, eBioscience), anti-CD3e (clone 500A2; BioLegend), anti-CD8a (clone 5H10; Caltag), anti-CD4 (clone RM4-5; BD Pharmingen), V450-conjugated CD45.1 (clone A20; BD Biosciences), PE-Cy7-conjugated anti-CD45.2 (clone 104; BD Biosciences), and anti-CD25 (clone PC61; BD Pharmingen). Functional grade anti-CD3 (clone 17A2) and anti-CD28 (clone 37.51) were purchased from eBioscience.

CTX assay. Blood (100-200 $\mu 1)$, obtained via submandibular vein, was allowed to clot for 1 hour at room temperature, and serum was collected by spinning down the cell pellet (1,000 $\mathrm{g}$ for 10 minutes). Serum was flash frozen and stored at $-80^{\circ} \mathrm{C}$. Serum CTX was measured using ELISA according to the manufacturer's instructions (Immunodiagnostic Systems)

Cytokine measurements. Peripheral blood $(200 \mu \mathrm{l})$ was obtained prior to sacrificing animals via mandible vein into EDTA-coated tubes (BD Biosciences). The blood was diluted 5-fold in PBS with $25 \mathrm{mM}$ EDTA and pelleted at $400 \mathrm{~g}$ for 10 minutes. The pellet was resuspended in $5 \mathrm{ml}$ red blood lysis media (PharmLyse; BD Biosciences) and incubated at room temperature for 5 minutes. Then, $15 \mathrm{ml}$ of PBS was added and repelleted. The pellets were resuspended in complete T cell media (RPMI, $10 \% \Delta \mathrm{FBS}$, penicillin-streptomycin-glutamine, nonessential amino acids, sodium pyruvate, HEPES, and $55 \mu \mathrm{M} \beta$-mercaptoethanol). Cells were plated on anti-CD3 $(1 \mu \mathrm{g} / \mathrm{ml})$ and anti-CD28 $(2 \mu \mathrm{g} / \mathrm{ml}$; both from eBioscience $)$ coated wells $\left(1 \times 10^{6}\right.$ to $3 \times 10^{6}$ cells/well; 24 well/plate in $\left.1 \mathrm{ml}\right)$ and incubated at $37^{\circ} \mathrm{C}$. After 30 hours, the plates were spun for 5 minutes at $300 \mathrm{~g}$, and media was collected, flash-frozen, and stored at $-80^{\circ} \mathrm{C}$. Cytokines in the media were quantitated using Luminex multiplexed ELISA system (Millipore) according to the manufacturers instructions. Six cytokines (IFN- $\gamma$, IL-1 $\beta$, IL-4, IL-6, TNF $\alpha$, and IL-17A) were measured, and only those that showed significant signal over background are shown in Figure 3.

$\mu C T$ data collection and analysis. The bones were scanned in $\mu \mathrm{CT} 40$ (Scanco Medical) at $55 \mathrm{kV}, 145$ $\mu \mathrm{A}$, and a resolution of $16 \mu \mathrm{m}$. Gaussian sigma of 1.2, Gaussian support of 2, lower threshold of 237, and upper threshold of 1,000 were used for all the analysis. Regions of interest were selected 50 slices below the growth plate of the proximal tibia to evaluate the trabecular compartment. Bone mineral density was obtained by quantitative $\mu \mathrm{CT}$ using Phantoms for calibration (53). All $\mu \mathrm{CT}$ data was collected and analyzed by JG, who was blinded to the treatment performed. 
Statistics. Statistical significance was assessed in all cases using paired 2-tailed Mann-Whitney $U$ test in GraphPad Prism 5.0. One-way and 2-way ANOVA was performed in GraphPad Prism 5.0.

Study approval. All animals were maintained in the Department of Comparative Medicine, St. Louis University School of Medicine, in accordance with institutional and Public Health Service Guidelines. St. Louis University School of Medicine IACUC approved all procedures performed on mice (protocol numbers 2072 and 2184).

\section{Author contributions}

ACS, ES, and ZSB performed the experiments. JG collected and analyzed $\mu$ CT data. DVN contributed to the design of experiments and data interpretation. RA designed and performed some experiments, as well as interpreted and wrote the manuscript with input from all coauthors.

\section{Acknowledgments}

The authors would like to thank Joy Eslick and Sheri Koehm in the Flow Core for their assistance with collecting FACS data. Research reported in this study was partially supported by National Institute of Arthritis and Musculoskeletal and Skin Disease of the NIH under R01AR064821 and R01AR068438. Washington University Musculoskeletal Research Core (NIH P30 AR057235) also partially supported this study.

Address correspondence to: Rajeev Aurora, 1100 S. Grand Boulevard DRC 605, St. Louis, Missouri 63104 , USA. Phone: 314.977.8891; E-mail: aurorar@slu.edu.

ZSB's current address is: Department of Radiation Oncology, Emory University School of Medicine, Atlanta, Georgia, USA.

1. Zaidi M. Skeletal remodeling in health and disease. Nat Med. 2007;13(7):791-801.

2. Dore RK. The RANKL pathway and denosumab. Rheum Dis Clin North Am. 2011;37(3):433-52.

3. Eghbali-Fatourechi G, Khosla S, Sanyal A, Boyle WJ, Lacey DL, Riggs BL. Role of RANK ligand in mediating increased bone resorption in early postmenopausal women. J Clin Invest. 2003;111(8):1221-1230.

4. Taxel P, Kaneko H, Lee SK, Aguila HL, Raisz LG, Lorenzo JA. Estradiol rapidly inhibits osteoclastogenesis and RANKL expression in bone marrow cultures in postmenopausal women: a pilot study. Osteoporos Int. 2008;19(2):193-199.

5. Krum SA, et al. Estrogen protects bone by inducing Fas ligand in osteoblasts to regulate osteoclast survival. EMBO J. 2008;27(3):535-545.

6. Nakamura T, et al. Estrogen prevents bone loss via estrogen receptor alpha and induction of Fas ligand in osteoclasts. Cell. 2007;130(5):811-823.

7. Park H, Jung YK, Park OJ, Lee YJ, Choi JY, Choi Y. Interaction of Fas ligand and Fas expressed on osteoclast precursors increases osteoclastogenesis. J Immunol. 2005;175(11):7193-7201.

8. Pacifici R. Role of T cells in ovariectomy induced bone loss--revisited. J Bone Miner Res. 2012;27(2):231-239.

9. Sato K, et al. Th17 functions as an osteoclastogenic helper T cell subset that links $\mathrm{T}$ cell activation and bone destruction. $J$ Exp Med. 2006;203(12):2673-2682.

10. Zhang YH, Heulsmann A, Tondravi MM, Mukherjee A, Abu-Amer Y. Tumor necrosis factor-alpha (TNF) stimulates RANKL-induced osteoclastogenesis via coupling of TNF type 1 receptor and RANK signaling pathways. J Biol Chem. 2001;276(1):563-568.

11. Zhao B, Grimes SN, Li S, Hu X, Ivashkiv LB. TNF-induced osteoclastogenesis and inflammatory bone resorption are inhibited by transcription factor RBP-J. J Exp Med. 2012;209(2):319-334.

12. Cenci S, et al. Estrogen deficiency induces bone loss by enhancing T-cell production of TNF-alpha. J Clin Invest. 2000;106(10):1229-1237.

13. Lam J, Takeshita S, Barker JE, Kanagawa O, Ross FP, Teitelbaum SL. TNF-alpha induces osteoclastogenesis by direct stimulation of macrophages exposed to permissive levels of RANK ligand. J Clin Invest. 2000;106(12):1481-1488.

14. Cummings SR, et al. Denosumab for prevention of fractures in postmenopausal women with osteoporosis. N Engl J Med. 2009;361(8):756-765.

15. Kiesel JR, Buchwald ZS, Aurora R. Cross-presentation by osteoclasts induces FoxP3 in CD8+ T cells. J Immunol. 2009;182(9):5477-5487.

16. Li H, Hong S, Qian J, Zheng Y, Yang J, Yi Q. Cross talk between the bone and immune systems: osteoclasts function as antigen-presenting cells and activate CD4+ and CD8+ T cells. Blood. 2010;116(2):210-217.

17. Mayer CT, Floess S, Baru AM, Lahl K, Huehn J, Sparwasser T. CD8+ Foxp3+ T cells share developmental and phenotypic features with classical CD4+ Foxp3+ regulatory T cells but lack potent suppressive activity. Eur J Immunol. 2011;41(3):716-725.

18. Abbas AK, et al. Regulatory T cells: recommendations to simplify the nomenclature. Nat Immunol. 2013;14(4):307-308.

19. Fontenot JD, Gavin MA, Rudensky AY. Foxp3 programs the development and function of CD4+CD25+ regulatory T cells. Nat Immunol. 2003;4(4):330-336.

20. Hori S, Sakaguchi S. Foxp3: a critical regulator of the development and function of regulatory T cells. Microbes Infect. 2004;6(8):745-751. 
21. Chatila TA. Role of regulatory T cells in human diseases. J Allergy Clin Immunol. 2005;116(5):949-59; quiz 960.

22. Kim JM, Rasmussen JP, Rudensky AY. Regulatory T cells prevent catastrophic autoimmunity throughout the lifespan of mice. Nat Immunol. 2007;8(2):191-197.

23. Sakaguchi S, Miyara M, Costantino CM, Hafler DA. FOXP3+ regulatory T cells in the human immune system. Nat Rev Immunol. 2010;10(7):490-500.

24. Blair PJ, Bultman SJ, Haas JC, Rouse BT, Wilkinson JE, Godfrey VL. CD4+CD8- T cells are the effector cells in disease pathogenesis in the scurfy (sf) mouse. J Immunol. 1994;153(8):3764-3774.

25. Buchwald ZS, Aurora R. Osteoclasts and CD8 T cells form a negative feedback loop that contributes to homeostasis of both the skeletal and immune systems. Clin Dev Immunol. 2013;2013:429373.

26. Buchwald ZS, Kiesel JR, Yang C, DiPaolo R, Novack DV, Aurora R. Osteoclast-induced Foxp3+ CD8 T-cells limit bone loss in mice. Bone. 2013;56(1):163-173.

27. Buchwald ZS, Kiesel JR, DiPaolo R, Pagadala MS, Aurora R. Osteoclast activated FoxP3+ CD8+ T-cells suppress bone resorption in vitro. PLoS One. 2012;7(6):e38199.

28. Henriksen K, et al. Degradation of the organic phase of bone by osteoclasts: a secondary role for lysosomal acidification. $J$ Bone Miner Res. 2006;21(1):58-66.

29. Hollberg K, Nordahl J, Hultenby K, Mengarelli-Widholm S, Andersson G, Reinholt FP. Polarization and secretion of cathepsin $\mathrm{K}$ precede tartrate-resistant acid phosphatase secretion to the ruffled border area during the activation of matrix-resorbing clasts. J Bone Miner Metab. 2005;23(6):441-449.

30. Brömme D, Lecaille F. Cathepsin K inhibitors for osteoporosis and potential off-target effects. Expert Opin Investig Drugs. 2009;18(5):585-600.

31. Li YP, Chen W. Characterization of mouse cathepsin K gene, the gene promoter, and the gene expression. J Bone Miner Res. 1999;14(4):487-499.

32. Leung P, Pickarski M, Zhuo Y, Masarachia PJ, Duong LT. The effects of the cathepsin K inhibitor odanacatib on osteoclastic bone resorption and vesicular trafficking. Bone. 2011;49(4):623-635.

33. Fuller $\mathrm{K}$, et al. Cathepsin $\mathrm{K}$ inhibitors prevent matrix-derived growth factor degradation by human osteoclasts. Bone. 2008;42(1):200-211.

34. Pennypacker BL, et al. Cathepsin K inhibitors prevent bone loss in estrogen-deficient rabbits. J Bone Miner Res. 2011;26(2):252-262

35. Kumar S, et al. A highly potent inhibitor of cathepsin $\mathrm{K}$ (relacatib) reduces biomarkers of bone resorption both in vitro and in an acute model of elevated bone turnover in vivo in monkeys. Bone. 2007;40(1):122-131.

36. Chen W, et al. Novel pycnodysostosis mouse model uncovers cathepsin $\mathrm{K}$ function as a potential regulator of osteoclast apoptosis and senescence. Hum Mol Genet. 2007;16(4):410-423.

37. Buchwald ZS, et al. A Bone Anabolic Effect of RANKL in a Murine Model of Osteoporosis Mediated Through FoxP3+ CD8 T Cells. J Bone Miner Res. 2015;30(8):1508-1522.

38. Daan NM, Fauser BC. Menopause prediction and potential implications. Maturitas. 2015;82(3):257-265.

39. Jee WS, Yao W. Overview: animal models of osteopenia and osteoporosis. J Musculoskelet Neuronal Interact. 2001;1(3):193-207.

40. Pacifici R. T cells and post menopausal osteoporosis in murine models. Arthritis Res Ther. 2007;9(2):102.

41. Li M, et al. Cell-associated ovalbumin is cross-presented much more efficiently than soluble ovalbumin in vivo. J Immunol. 2001;166(10):6099-6103.

42. Clarke SR, Barnden M, Kurts C, Carbone FR, Miller JF, Heath WR. Characterization of the ovalbumin-specific TCR transgenic line OT-I: MHC elements for positive and negative selection. Immunol Cell Biol. 2000;78(2):110-117.

43. Weitzmann MN, Pacifici R. The role of T lymphocytes in bone metabolism. Immunol Rev. 2005;208:154-168.

44. Jilka RL. Molecular and cellular mechanisms of the anabolic effect of intermittent PTH. Bone. 2007;40(6):1434-1446.

45. Yamane H, Sakai A, Mori T, Tanaka S, Moridera K, Nakamura T. The anabolic action of intermittent PTH in combination with cathepsin $\mathrm{K}$ inhibitor or alendronate differs depending on the remodeling status in bone in ovariectomized mice. Bone. 2009;44(6):1055-1062.

46. Wu X, McKenna MA, Feng X, Nagy TR, McDonald JM. Osteoclast apoptosis: the role of Fas in vivo and in vitro. Endocrinology. 2003;144(12):5545-5555.

47. Zhao R, Wang X, Feng F. Upregulated Cellular Expression of IL-17 by CD4+ T-Cells in Osteoporotic Postmenopausal Women. Ann Nutr Metab. 2016;68(2):113-118.

48. Schreiweis MA, et al. A proteomic analysis of adult rat bone reveals the presence of cartilage/chondrocyte markers. J Cell Biochem. 2007;101(2):466-476.

49. Komatsu N, et al. Pathogenic conversion of Foxp3+ T cells into TH17 cells in autoimmune arthritis. Nat Med. 2014;20(1):62-68.

50. Zhang HG, et al. Depletion of collagen II-reactive T cells and blocking of B cell activation prevents collagen II-induced arthritis in DBA/1j mice. J Immunol. 2002;168(8):4164-4172.

51. Tada Y, Ho A, Koh DR, Mak TW. Collagen-induced arthritis in CD4- or CD8-deficient mice: CD8+ T cells play a role in initiation and regulate recovery phase of collagen-induced arthritis. J Immunol. 1996;156(11):4520-4526.

52. Haque MA, et al. Evidence for immune responses to a self-antigen in lung transplantation: role of type V collagen-specific $\mathrm{T}$ cells in the pathogenesis of lung allograft rejection. J Immunol. 2002;169(3):1542-1549.

53. Nazarian A, Snyder BD, Zurakowski D, Müller R. Quantitative micro-computed tomography: a non-invasive method to assess equivalent bone mineral density. Bone. 2008;43(2):302-311. 